\title{
A KEPLERIAN CIRCUMBINARY DISK AROUND THE PROTOSTELLAR SYSTEM L1551 NE
}

\author{
Shigehisa Takakuwa ${ }^{1}$, Masao Saito ${ }^{2}$, Jeremy Lim $^{3}$, Kazuya Saigo ${ }^{2}$, T. K. Sridharan ${ }^{4}$, And Nimesh A. Patel ${ }^{4}$ \\ ${ }^{1}$ Academia Sinica Institute of Astronomy and Astrophysics, P.O. Box 23-141, Taipei 10617, Taiwan; takakuwa@ asiaa.sinica.edu.tw \\ ${ }^{2}$ ALMA Project Office, National Astronomical Observatory of Japan, Osawa 2-21-1, Mitaka, Tokyo 181-8588, Japan \\ ${ }^{3}$ Department of Physics, University of Hong Kong, Pokfulam Road, Hong Kong \\ ${ }^{4}$ Harvard-Smithsonian Center for Astrophysics, 60 Garden Street, MS78, Cambridge, MA 02138, USA \\ Received 2011 October 25; accepted 2012 May 16; published 2012 July 5
}

\begin{abstract}
We present Submillimeter Array observations of a Keplerian disk around the Class I protobinary system L1551 $\mathrm{NE}$ in $335 \mathrm{GHz}$ continuum emission and submillimeter line emission in ${ }^{13} \mathrm{CO}(J=3-2)$ and $\mathrm{C}^{18} \mathrm{O}(J=3-2)$ at a resolution of $\sim 120 \times 80 \mathrm{AU}$. The $335 \mathrm{GHz}$ dust-continuum image shows a strong central peak closely coincident with the binary protostars and likely corresponding to circumstellar disks, surrounded by a $\sim 600 \times 300$ AU feature elongated approximately perpendicular to the $[\mathrm{Fe}$ II] jet from the southern protostellar component suggestive of a circumbinary disk. The ${ }^{13} \mathrm{CO}$ and $\mathrm{C}^{18} \mathrm{O}$ images confirm that the circumbinary continuum feature is indeed a rotating disk; furthermore, the $\mathrm{C}^{18} \mathrm{O}$ channel maps can be well modeled by a geometrically thin disk exhibiting Keplerian rotation. We estimate a mass for the circumbinary disk of $\sim 0.03-0.12 M_{\odot}$, compared with an enclosed mass of $\sim 0.8 M_{\odot}$ that is dominated by the protobinary system. Compared with several other Class I protostars known to exhibit Keplerian disks, L1551 NE has the lowest bolometric temperature ( $\sim 91 \mathrm{~K})$, highest envelope mass $\left(\sim 0.39 M_{\odot}\right)$, and the lowest ratio in stellar mass to envelope + disk + stellar mass $(\sim 0.65)$. L1551 NE may therefore be the youngest protostellar object so far found to exhibit a Keplerian disk. Our observations present firm evidence that Keplerian disks around binary protostellar systems, "Keplerian circumbinary disks," can exist. We speculate that tidal effects from binary companions could transport angular momenta toward the inner edge of the circumbinary disk and create the Keplerian circumbinary disk.
\end{abstract}

Key words: ISM: individual objects (L1551 NE) - ISM: molecules - stars: formation

\section{INTRODUCTION}

Previous interferometric observations of millimeter and submillimeter continuum emission have shown compact $(\lesssim 600 \mathrm{AU})$ components around both Class 0 and I protostars, embedded in extended ( $\gtrsim 2000 \mathrm{AU}$ ) envelopes (Looney et al. 2000; Harvey et al. 2003a, 2003b; Enoch et al. 2009, 2011; Jørgensen et al. 2009; Maury et al. 2010). Subarcsecondresolution continuum observations that resolve the compact components reveal flattened morphologies commonly attributed to circumstellar or possibly circumbinary disks (Looney et al. 2000; Brinch et al. 2007; Enoch et al. 2009, 2011). The masses of the putative circumstellar disks around Class 0 and I protostars are on the order of $0.01-1.0 M_{\odot}$, much higher than the typical masses of circumstellar disks around Class II (T Tauri) stars $\left(\sim 0.005 M_{\odot}\right.$; Andrews \& Williams 2005,2007$)$. The sizes of the circumstellar disks $(\sim 600 \mathrm{AU})$ around all these classes of stars, however, are comparable (Guilloteau \& Dutrey 1998; Guilloteau et al. 1999; Schaefer et al. 2009).

Previous interferometric observations in molecular lines have shown an infalling motion and/or little rotating motion in circumstellar disks around Class 0 protostars (Takakuwa et al. 2007; Brinch et al. 2009; Yen et al. 2010, 2011). Such "pseudodisks" have been predicted by theoretical simulations to form in the inner regions of envelopes around protostars (Mellon \& Li 2008, 2009; Vorobyov 2009; Machida et al. 2010, 2011; Machida \& Matsumoto 2011). On the other hand, interferometric observations of $\mathrm{T}$ Tauri stars in millimeter molecular lines have revealed Keplerian disks around Class II stars (Guilloteau \& Dutrey 1998; Guilloteau et al. 1999; Piétu et al. 2003; Schaefer et al. 2009). These studies suggest that the transition from pseudo-disks to Keplerian disks occurs sometime between the Class 0 and II stages.
Interferometric observations of molecular lines with, especially, the Submillimeter Array (SMA), which can (better) image higher transitions that trace gas at higher densities and/or temperatures, have been increasingly successful at finding Keplerian disks around Class I protostars. Brinch et al. (2007) have identified an (radius) $r \sim 200 \mathrm{AU}$ scale Keplerian disk around L1489 IRS with a central stellar mass of $1.35 M_{\odot}$. Observations by Lommen et al. (2008) have also revealed an $r \sim 100 \mathrm{AU}$ scale Keplerian disk with a central stellar mass of $0.37 M_{\odot}$ around IRS 63 , and an $r \sim 200 \mathrm{AU}$ scale Keplerian disk with a central stellar mass of $2.5 M_{\odot}$ around Elias 29. Observations by Jørgensen et al. (2009) have shown an $r \sim 140$ AU scale Keplerian disk around the Class I protostar IRS 43 with a central stellar mass of $1.0 M_{\odot}$. The masses of these Keplerian disks range from $\sim 0.004$ to $\sim 0.06 M_{\odot}$, slightly larger than that around T Tauri stars, whereas their radii are comparable to those of the Keplerian disks observed around T Tauri stars.

Current theoretical simulations have trouble transforming pseudo-disks to Keplerian disks around protostars. Mellon \& Li $(2008,2009)$ have performed ideal magnetohydrodynamic (MHD) simulations of self-similar, collapsing envelopes to investigate formation of Keplerian disks. They found that, for typical magnetic field strengths in interstellar clouds, magnetic braking is so efficient that centrifugally supported disks cannot form. Machida et al. $(2010,2011)$ and Machida \& Matsumoto (2011) have conducted three-dimensional MHD simulations of a collapsing Bonnor-Ebert sphere, including non-ideal MHD effects and finite spherical radii $\left(R_{\mathrm{c}}=6.2 \times 10^{3} \mathrm{AU}\right.$ for a mass of $\left.1.0 M_{\odot}\right)$. Their models show that the Ohmic dissipation and the depletion of the surrounding infalling envelope that anchors the magnetic field significantly reduce the effect of the magnetic field on the disk formation. The predicted masses of the disks, however, are $\sim$ a few to $\sim$ a few hundred times higher than the masses of the central protostar, and hence the disks are subject 
to further fragmentation. Thus further long-term simulations are required to reproduce the observed Keplerian disks around the Class I protostars. To provide observational insights to theoretical models, it is important to identify a Keplerian disk just after the transition from the pseudo-disk, and to compare its properties to those of previously identified Keplerian disks and theoretical models.

Millimeter continuum observations have also revealed evidence for disks around binary protostars, "circumbinary disks" (Looney et al. 1997, 2000; Moriarty-Schieven et al. 2000; Lim \& Takakuwa 2006; Maury et al. 2010; Chen et al. 2012). It is intriguing to investigate whether Keplerian rotation is present in those circumbinary disks as well as disks around single protostars. Although the Class I protostars around which Keplerian disks have been found are thought to be single, it is possible that many and perhaps all are binary (or multiple) given the high frequency of binarity around pre-main-sequence and main-sequence solar-mass stars (Duquennoy \& Mayor 1991; Delfosse et al. 1999; Mathieu et al. 2000), as has been suggested for L1489 IRS (Hogerheijde \& Sandell 2000; Brinch et al. 2007). It is therefore important to compare the properties of circumbinary disks around known closely separated binary protostellar systems with the presumed circumstellar disks of apparently single protostars.

In this paper, we report observations of the Class I protostar L1551 NE in the $335 \mathrm{GHz}$ continuum emission, along with the ${ }^{13} \mathrm{CO}(J=3-2)$ and $\mathrm{C}^{18} \mathrm{O}(J=3-2)$ lines, at subarcsecond resolution. Compared to the abovementioned Class I sources associated with the Keplerian disks, L1551 NE is likely the youngest Class I object, since the bolometric temperature $\left(T_{\text {bol }}=91 \mathrm{~K}\right.$; Froebrich 2005$)$ is more than a factor of three lower and the amount of the surrounding envelope around L1551 $\mathrm{NE}$ is $\sim$ one order of magnitude higher than that around the other protostars (Yokogawa et al. 2003; Moriarty-Schieven et al. 2006; Takakuwa \& Kamazaki 2011). L1551 NE contains two $3.6 \mathrm{~cm}$ radio continuum sources with a projected separation of $\sim 70 \mathrm{AU}$ at a position angle of $\sim 300^{\circ}$ : the southeastern source is referred to as "Source A" and the northwestern source "Source B" (Reipurth et al. 2002). Near-infrared observations of L1551 NE have revealed that Source A drives collimated [Fe II] jets along the northeast to southwest direction at a position angle of $\sim 60^{\circ}$ (Reipurth et al. 2000, 2002; Hayashi \& Pyo 2009), and Source B is located at the origin of an extended ( 2000 AU) NIR reflection nebula (Reipurth et al. 2000, 2002; Hayashi \& Pyo 2009). There is little doubt that L1551 NE is a binary protostellar system. Our observations of this nearby ( $d=140 \mathrm{pc}$; Elias 1978$)$ object reveal for the first time a Keplerian circumbinary disk. In the following, we shall describe observations (Section 2), continuum and molecularline results (Section 3), and the analysis to identify a Keplerian disk (Section 4). In the last section (Section 5), we will compare the properties of the Keplerian disk around L1551 NE to those around the other protostars and discuss the evolution and the formation mechanism of circumstellar/Keplerian circumbinary disks.

\section{SMA OBSERVATIONS}

We observed L1551 NE with the extended configuration of the $\mathrm{SMA}^{5}$ on 2010 January 15 and 18 . The ${ }^{13} \mathrm{CO}(J=3-2$;

\footnotetext{
5 The SMA is a joint project between the Smithsonian Astrophysical Observatory and the Academia Sinica Institute of Astronomy and Astrophysics and is funded by the Smithsonian Institution and the Academia Sinica.
}

Table 1

Parameters for the SMA Observations of L1551 NE

\begin{tabular}{|c|c|c|}
\hline \multirow[t]{2}{*}{ Parameter } & \multicolumn{2}{|c|}{ Value } \\
\hline & 2010 January 15 & 2010 January 18 \\
\hline Number of antennas & 8 & 7 \\
\hline Right ascension (J2000.0) & \multicolumn{2}{|c|}{$04^{\mathrm{h}} 31^{\mathrm{m}} 44^{\mathrm{s}} \cdot 47$} \\
\hline Declination (J2000.0) & \multicolumn{2}{|c|}{$18^{\circ} 08^{\prime} 32^{\prime \prime} .2$} \\
\hline Primary beam HPBW & \multicolumn{2}{|c|}{$\sim 37^{\prime \prime}$} \\
\hline Synthesized beam HPBW (line) & \multicolumn{2}{|c|}{$0^{\prime} .95 \times 0.0^{\prime} .66\left(\right.$ P.A. $\left.=-88^{\circ}\right)$} \\
\hline Synthesized beam HPBW (continuum) & \multicolumn{2}{|c|}{$0^{\prime} .80 \times 0^{\prime \prime} .54\left(\right.$ P.A. $\left.=-87^{\circ}\right)$} \\
\hline Baseline coverage & \multicolumn{2}{|c|}{$29-249(\mathrm{k} \lambda)$} \\
\hline Conversion factor $\left(\mathrm{C}^{18} \mathrm{O}\right)$ & \multicolumn{2}{|c|}{$1\left(\mathrm{Jy} \mathrm{beam}^{-1}\right)=17.9(\mathrm{~K})$} \\
\hline Frequency resolution & \multicolumn{2}{|c|}{$406.25 \mathrm{kHz} \sim 0.37 \mathrm{~km} \mathrm{~s}^{-1}$} \\
\hline Bandwidth & \multicolumn{2}{|c|}{$7.74 \mathrm{GHz}$} \\
\hline Flux calibrator & Titan & Callisto \\
\hline Gain calibrator & \multicolumn{2}{|c|}{$0423-013,3 \mathrm{c} 120$} \\
\hline Flux (0423-013 upper) & $3.46 \mathrm{Jy}$ & $3.62 \mathrm{Jy}$ \\
\hline Flux (0423-013 lower) & $3.53 \mathrm{Jy}$ & $4.42 \mathrm{Jy}$ \\
\hline Flux (3c120 upper) & $0.80 \mathrm{Jy}$ & $0.92 \mathrm{Jy}$ \\
\hline Flux (3c120 lower) & $0.83 \mathrm{Jy}$ & $1.08 \mathrm{Jy}$ \\
\hline Passband calibrator & \multicolumn{2}{|c|}{$3 c 273$} \\
\hline System temperature (DSB) & $\sim 300-700 \mathrm{~K}$ & $\sim 400-1000 \mathrm{~K}$ \\
\hline rms noise level (continuum) & \multicolumn{2}{|c|}{$3 \mathrm{mJy}$ beam $^{-1}$} \\
\hline rms noise level $\left(\mathrm{C}^{18} \mathrm{O}\right)$ & \multicolumn{2}{|c|}{$0.117 \mathrm{Jy}_{\text {beam }^{-1}}$} \\
\hline rms noise level $\left({ }^{13} \mathrm{CO}\right)$ & \multicolumn{2}{|c|}{$0.107 \mathrm{Jy}_{\text {beam }}-1$} \\
\hline
\end{tabular}

$330.587965 \mathrm{GHz})$ and $\mathrm{C}^{18} \mathrm{O}(J=3-2 ; 329.3305453 \mathrm{GHz})$ lines, and the continuum emission at both the upper and lower sidebands, were measured simultaneously. The spectral windows ("chunks") of the SMA correlator assigned to the ${ }^{13} \mathrm{CO}$ and $\mathrm{C}^{18} \mathrm{O}$ lines had a resolution of $406.25 \mathrm{kHz}$, corresponding to a velocity resolution of $\sim 0.37 \mathrm{~km} \mathrm{~s}^{-1}$. In each sideband there are a total of 48 chunks, providing a total bandwidth of $4 \mathrm{GHz}$. All the chunks at both sidebands except for those assigned for the ${ }^{13} \mathrm{CO}$ and $\mathrm{C}^{18} \mathrm{O}$ lines were combined to make a single continuum channel. The frequency coverages of the continuum observation were $327.16-329.24 \mathrm{GHz}(\lambda=0.911-0.916 \mathrm{~mm})$, 329.34-330.47 GHz (0.907-0.910 mm), 330.57-331.13 GHz (0.905-0.907 mm), and 339.16-343.13 GHz (0.874-0.884 mm). The total bandwidth of the continuum observation was 7.74 GHz. In the following, we refer to the continuum emission taken with the present SMA observations as "335 GHz continuum emission," since the mean frequency of the combined continuum data is $\sim 335.4 \mathrm{GHz}$. Details of the SMA are described by Ho et al. (2004). Table 1 summarizes the observational parameters. The minimum projected baseline length was $\sim 29 \mathrm{k} \lambda$ at the $\mathrm{C}^{18} \mathrm{O}$ frequency, and for a Gaussian emission distribution with an FWHM of $\sim 7^{\prime \prime}(\sim 1000 \mathrm{AU})$, the peak flux recovered is $\sim 10 \%$ of the peak flux of the Gaussian (Wilner $\&$ Welch 1994). The estimated uncertainty in the absolute flux calibration is $\sim 30 \%$. The raw visibility data were calibrated and flagged with MIR, which is an IDL-based data reduction package (Scoville et al. 1993). The calibrated visibility data were Fourier-transformed and CLEANed with MIRIAD to produce the images (Sault et al. 1995).

\section{RESULTS}

\section{1. $335 \mathrm{GHz}$ Continuum Emission}

Figure 1 shows the $335 \mathrm{GHz}$ continuum image of L1551 $\mathrm{NE}$ at an angular resolution of $00^{\prime} 80 \times 0.54$ (P.A. $=-87^{\circ}$ ), corresponding to a spatial resolution of $110 \times 80 \mathrm{AU}$. The submillimeter continuum emission exhibits three distinct peaks. 


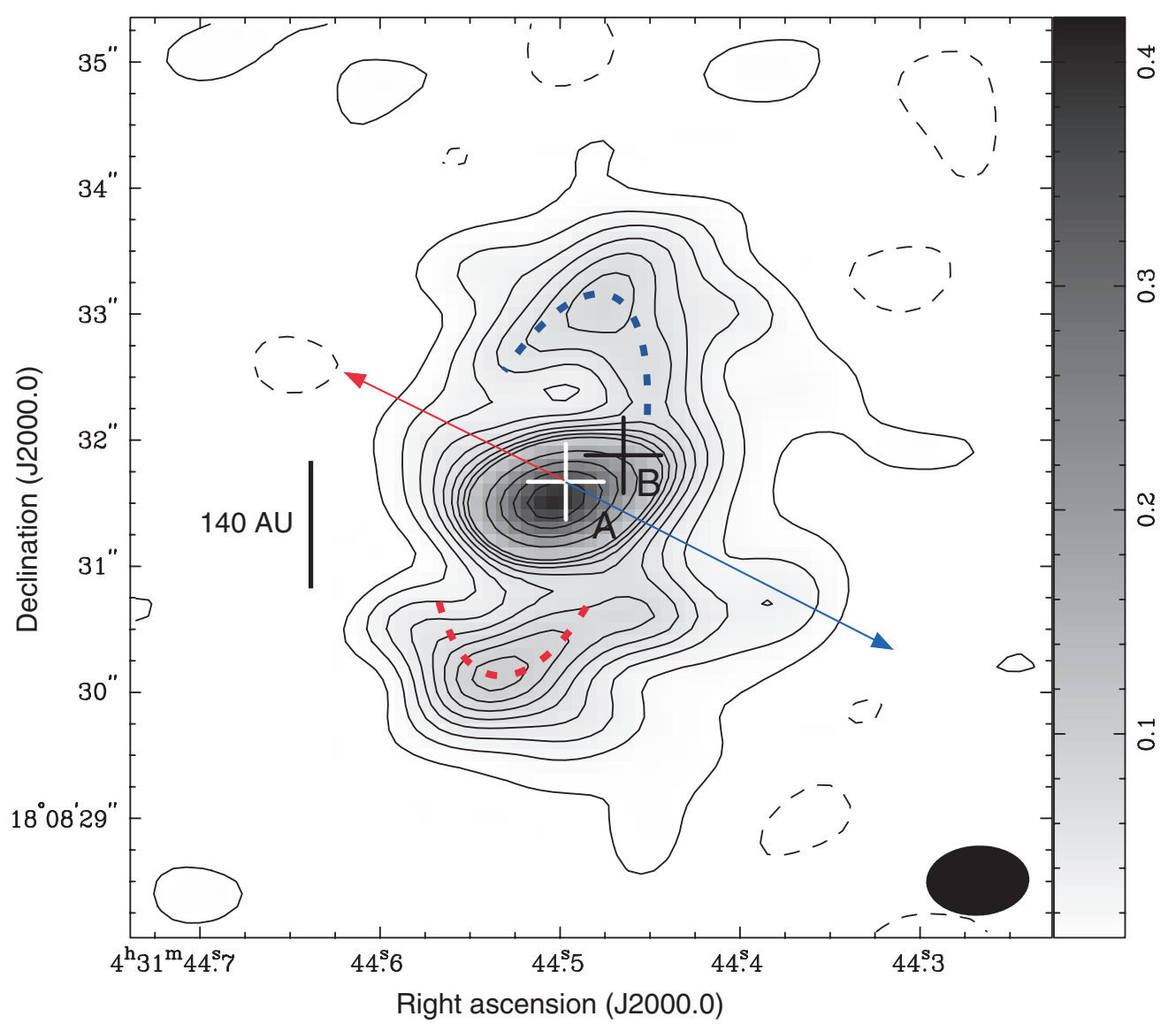

Figure 1. $335 \mathrm{GHz}$ continuum image of L1551 NE observed with the SMA. Contour levels are from $3 \sigma$ in steps of $4 \sigma$ until $35 \sigma$, and then in steps of $20 \sigma$ (1 $\sigma=$ $3 \mathrm{mJy}_{\text {beam }}^{-1}$ ). The highest contour level is $115 \sigma$. Crosses indicate the positions of Sources A and B as labeled. A filled ellipse at the bottom-right corner shows the synthesized beam $\left(0^{\prime \prime} 80 \times 0^{\prime \prime} 54\right.$; P.A. $\left.=-87^{\circ}\right)$. Blue and red dashed curves delineate the tilted $U$-shaped features, and blue and red arrows show the direction of the blueshifted and redshifted [Fe II] jets driven by Source A.

The bright central peak is located $\sim 30$ AU SE from Source A, and exhibits a marginal extension toward Source B (note that the central peak is elongated in a direction that is different from that of the synthesized beam). This central component appears to be closely coincident with the protobinary. From the two-dimensional Gaussian fitting, the total flux density of this component is measured to be $\sim 0.73 \mathrm{Jy}$ and the deconvolved size to be $0^{\prime} .90 \times 00^{\prime} .49$ (P.A. $\left.=-72^{\circ}\right)$. The other two peaks, one to the north and the other to the south, are located almost symmetrically with respect to the central component. These peaks exhibit tilted $U$-shaped morphologies (see dashed curves in Figure 1), which trace a (diameter) $D \sim 600 \times 300 \mathrm{AU}$ scale inclined ring-like feature. The position angle of this feature $\left(\sim 160^{\circ}\right)$ is approximately perpendicular to the axis of the [Fe II] jets driven by Source A (P.A. $=64^{\circ}$; see blue and red arrows in Figure 1). The total flux density integrated over the entire emission region including these three components is $\sim 1.65 \mathrm{Jy}$. A similar three-peak feature, albeit at a lower signal-to-noise ratio $(\mathrm{S} / \mathrm{N})$, is also seen in the $1.3 \mathrm{~mm}$ continuum image of L1551 NE taken with the OVRO (Moriarty-Schieven et al. 2000). Without the benefit of observations in molecular lines, however, the interpretation made by Moriarty-Schieven et al. (2000) for the continuum structure is very different from ours as described in Section 4.

Single-dish observations of L1551 NE in the $850 \mu \mathrm{m}$ continuum show an extended $\left(=9{ }^{\prime} .8 \times 8^{\prime \prime} .8\right)$ envelope with a total flux density of $2.78 \mathrm{Jy}$ (Moriarty-Schieven et al. 2006). To assess the contribution from the extended envelope to the more com- pact emission observed with the SMA, we created a 9'. $8 \times 8^{\prime \prime} .8$ Gaussian image with a total flux density of $2.78 \mathrm{Jy}$, and sampled the emission with the $u v$-coverage of our SMA observations. We found that the envelope is almost entirely resolved out: We recovered a flux density of only $\sim 7.6 \mathrm{mJy}$, less than $0.5 \%$ of the total flux density of the more compact emission observed with the SMA. Thus, the contribution from the extended envelope to the more compact structure observed with the SMA is likely negligible. Similarly, Jørgensen et al. (2009) found that the contribution from extended envelope emission measured at $\mathrm{mm} / \mathrm{sub}-\mathrm{mm}$ wavelengths in single-dish observations to that in SMA observations having baselines $\geqslant 50 k \lambda$ is at most $8 \%$ for various envelope models. We estimated a total (gas + dust) mass $\left(\equiv M_{g+d}\right)$ for the feature detected in the $335 \mathrm{GHz}$ continuum as shown in Figure 1 of

$$
M_{g+d}=\frac{S_{\nu} d^{2}}{\kappa_{\nu} B_{v}\left(T_{d}\right)},
$$

where $v$ is the frequency, $S_{v}$ is the flux density, $d$ is the distance, $B_{v}\left(T_{d}\right)$ is the Planck function, $T_{d}$ is the dust temperature, and $\kappa_{v}$ is the dust opacity per unit gas + dust mass on the assumption that the gas-to-dust mass ratio is 100 . The frequency dependence of $\kappa_{\nu}$ can be expressed as $\kappa_{\nu}=\kappa_{\nu_{0}}\left(\nu / \nu_{0}\right)^{\beta}$, where $\beta$ denotes the dust-opacity index. By modeling the spectral energy distribution (SED) of the envelope as measured in single-dish observations at wavelengths from $12 \mu \mathrm{m}$ to $2 \mathrm{~mm}$, Moriarty-Schieven et al. (1994) derived $\beta \sim 1$, and Barsony \& Chandler (1993) and 


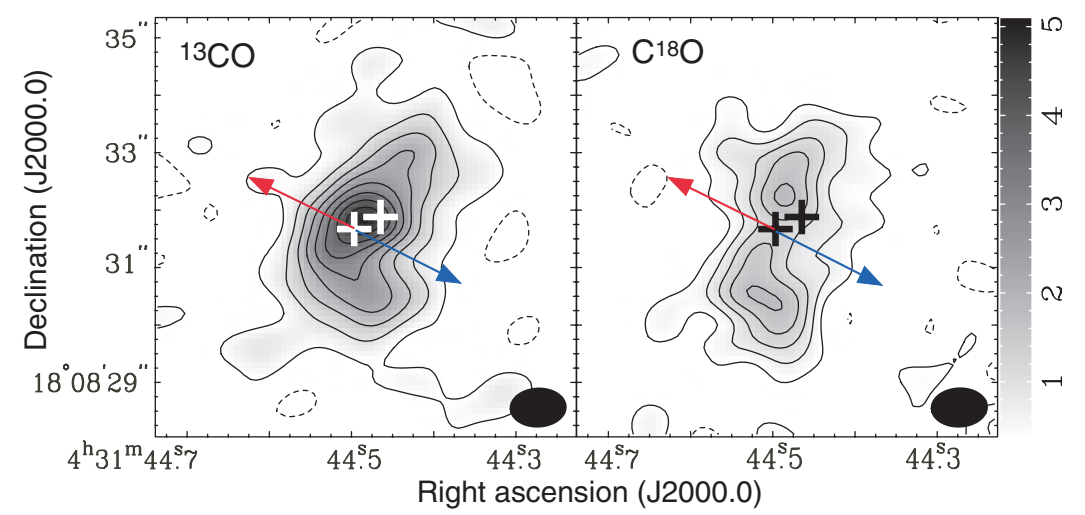

Figure 2. Moment 0 maps of the ${ }^{13} \mathrm{CO}(3-2)$ (left) and $\mathrm{C}^{18} \mathrm{O}(3-2)$ lines (right) in L1551 NE observed with the SMA. In the ${ }^{13} \mathrm{CO}$ map, contour levels are from $2 \sigma$ in steps of $3 \sigma\left(1 \sigma=0.189 \mathrm{Jy}_{\text {beam }}^{-1} \mathrm{~km} \mathrm{~s}^{-1}\right)$, and the integrated-velocity range is $2.28-10.76 \mathrm{~km} \mathrm{~s}^{-1}$. In the $\mathrm{C}^{18} \mathrm{O}$ map, contour levels are from $2 \sigma$ in steps of $2 \sigma$ $\left(1 \sigma=0.173 \mathrm{Jy} \mathrm{beam}^{-1} \mathrm{~km} \mathrm{~s}^{-1}\right)$, and the integrated-velocity range is $3.72-9.63 \mathrm{~km} \mathrm{~s}^{-1}$. Crosses indicate the positions of the protobinary, and filled ellipses at the bottom-right corner indicate the synthesized beam $\left(0^{\prime} \cdot 95 \times 0^{\prime \prime} 66 ;\right.$ P.A. $\left.=-88^{\circ}\right)$. Blue and red arrows show the direction of the blueshifted and redshifted $[$ Fe II] jets driven by Source A. A right-hand scale bar shows the intensities of the gray-scale images in Jy beam ${ }^{-1} \mathrm{~km} \mathrm{~s}^{-1}$.

Moriarty-Schieven et al. (1994) derived $T_{d} \sim 42 \mathrm{~K}$. If we adopt $\kappa_{\nu_{0}}=\kappa_{1200 \mathrm{GHz}}=0.1 \mathrm{~cm}^{2} \mathrm{~g}^{-1}$ (Beckwith et al. 1990), then from Equation (1) we find $M_{g+d} \sim 0.047 M_{\odot}$. This value is comparable with that estimated by Moriarty-Schieven et al. (2000) from their OVRO $1.3 \mathrm{~mm}$ image $\left(\sim 0.060 M_{\odot}\right)$ based on the same parameters but after correcting for their different adopted distance of $\sim 160$ pc. Jørgensen et al. (2009) performed a survey of low-mass protostars with the SMA and adopted the dust opacity of Ossenkopf \& Henning (1994) for grains with thin ice mantles coagulated at a density of $n_{\mathrm{H}_{2}} \sim 10^{6}$ $\mathrm{cm}^{-3}$, i.e., $\kappa_{850} \mu \mathrm{m}=0.0182 \mathrm{~cm}^{2} \mathrm{~g}^{-1}$ and $\beta=1.7$. To permit a more direct comparison with the results of Jørgensen et al. (2009), we adopt this dust opacity law and $T_{d}=42 \mathrm{~K}$, and from Equation (1) we find $M_{g+d} \sim 0.078 M_{\odot}$. Jørgensen et al. (2009) referred to simulations by Visser et al. (2009) that showed dust temperatures at $r=200 \mathrm{AU}$ ranging from $\sim 20$ to $50 \mathrm{~K}$. This range in dust temperatures gives $M_{g+d}=0.063 M_{\odot}\left(T_{d}=\right.$ $50 \mathrm{~K})-0.21 M_{\odot}\left(T_{d}=20 \mathrm{~K}\right)$ with the same dust opacity law as Ossenkopf \& Henning (1994). These estimates show that $M_{g+d}$ is probably within the range of $\sim 0.05-0.2 M_{\odot}$. The same methods and the flux density of the central component derived from the two-dimensional Gaussian fitting $(\sim 0.73 \mathrm{Jy})$ give the mass of the central component of $\sim 0.02-0.09 M_{\odot}$. Subtraction of this mass from $M_{g+d}$ gives the mass of the rest of the emission components, $\sim 0.03-0.12 M_{\odot}$.

$$
\text { 3.2. }{ }^{13} \mathrm{CO}(3-2) \text { and } \mathrm{C}^{18} \mathrm{O}(3-2)
$$

Figure 2 shows integrated intensity maps in ${ }^{13} \mathrm{CO}(3-2)$ and $\mathrm{C}^{18} \mathrm{O}(3-2)$. In both ${ }^{13} \mathrm{CO}$ and $\mathrm{C}^{18} \mathrm{O}$, the emission is elongated along the northwest to southeast direction. This elongation is approximately perpendicular to the axis of the [Fe II] jets driven by Source A, just like the ring-like structure seen in the continuum. Furthermore, just like this structure, the emission in both lines has outer dimensions of $\sim 600 \times 300$ AU. Although the overall sizes and position angles of the major axes of molecular and ring-like continuum structures are similar, their internal structures are quite different. The three peaks seen in the continuum emission are not evident in ${ }^{13} \mathrm{CO}$ and $\mathrm{C}^{18} \mathrm{O}$. Instead, the ${ }^{13} \mathrm{CO}$ emission exhibits a single peak between Sources A and B ( $\sim 40$ AU NW from Source A), whereas the bright central peak in the continuum emission is located $\sim 30$ AU SE from Source A. The positional accuracy of the ${ }^{13} \mathrm{CO}$ emission peak can be approximately estimated as $\sim$ beam size $/ \mathrm{S} / \mathrm{N} \lesssim 5 \mathrm{AU}$, and that of the continuum emission peak $\lesssim 1$ AU. Thus, the positional offsets of the ${ }^{13} \mathrm{CO}$ emission peak from the continuum peak and the position of Source A are statistically significant. By contrast, the $\mathrm{C}^{18} \mathrm{O}$ emission does not show a central peak, but instead shows two peaks at $\sim 80$ AU NW and $\sim 170$ AU SE from Source A, closer in than the two outer continuum peaks which are located $\sim 210$ AU NW and SE from Source A. The positional accuracy of these two $\mathrm{C}^{18} \mathrm{O}$ emission peaks is estimated to be $\lesssim 13 \mathrm{AU}$, and that of the two continuum peaks $\lesssim 5$ AU. Therefore, the offsets of the two $\mathrm{C}^{18} \mathrm{O}$ emission peaks from the outer continuum peaks are also real. The different emission distributions are likely due to the combination of different amounts of envelope (and, in molecular lines, possibly also ambient) emission recovered, differences in optical depths, and molecular abundance variations; these different effects are difficult to disentangle with the available data.

Figures 3 and 4 show velocity channel maps of the ${ }^{13} \mathrm{CO}$ and $\mathrm{C}^{18} \mathrm{O}$ lines, respectively. Around $V_{\mathrm{LSR}} \sim 7 \mathrm{~km} \mathrm{~s}^{-1}$ both the ${ }^{13} \mathrm{CO}$ and $\mathrm{C}^{18} \mathrm{O}$ emission are significantly suppressed, a likely effect of missing flux at the ambient velocity of the molecular gas immediately around L1551 NE. Indeed, singledish CS (3-2, 5-4, 7-6) spectra toward L1551 NE show a central velocity of $V_{\mathrm{LSR}} \sim 7 \mathrm{~km} \mathrm{~s}^{-1}$ (Moriarty-Schieven et al. 1995b). A single-dish $\mathrm{C}^{18} \mathrm{O}$ (3-2) spectrum taken toward L1551 $\mathrm{NE}$ with the CSO shows two distinct components: one having a narrower velocity width $\left(\sim 0.68 \mathrm{~km} \mathrm{~s}^{-1}\right)$ with a central velocity of $V_{\mathrm{LSR}} \sim 6.7 \mathrm{~km} \mathrm{~s}^{-1}$, and the other having a broader velocity width $\left(\sim 2.2 \mathrm{~km} \mathrm{~s}^{-1}\right)$ with a central velocity of $V_{\mathrm{LSR}} \sim$ $7.0 \mathrm{~km} \mathrm{~s}^{-1}$ (Fuller \& Ladd 2002). The velocity range and the central velocity of the broader component are similar to those of the $\mathrm{C}^{18} \mathrm{O}$ emission observed with the SMA. We therefore adopt a systemic velocity for L1551 NE of $V_{\mathrm{LSR}}=7 \mathrm{~km} \mathrm{~s}^{-1}$. In the ${ }^{13} \mathrm{CO}$ velocity channel maps, there are high-velocity blueshifted (2.5-3.6 $\left.\mathrm{km} \mathrm{s}^{-1}\right)$ and redshifted $\left(9.8-10.2 \mathrm{~km} \mathrm{~s}^{-1}\right.$ ) components just displaced to the west and east of Source A, respectively. These high-velocity ${ }^{13} \mathrm{CO}$ components are not detected in the $\mathrm{C}^{18} \mathrm{O}$ emission. In the lower-blueshifted velocity $\left(\sim 3.9-5.8 \mathrm{~km} \mathrm{~s}^{-1}\right)$, both the ${ }^{13} \mathrm{CO}$ and $\mathrm{C}^{18} \mathrm{O}$ emission are located predominantly to the north of the protobinary, and in the lower-redshifted velocity $\left(\sim 7.6 \mathrm{~km} \mathrm{~s}^{-1}-9.5 \mathrm{~km} \mathrm{~s}^{-1}\right)$ to the south of the protobinary. Furthermore, the extents of the ${ }^{13} \mathrm{CO}$ and $\mathrm{C}^{18} \mathrm{O}$ emission appear to become progressively larger as the velocity approaches the systemic velocity. A comparison of the SMA $\mathrm{C}^{18} \mathrm{O}(3-2)$ integrated spectrum with the CSO $\mathrm{C}^{18} \mathrm{O}$ (3-2) spectrum shows that from $\sim 3.9$ to $5.4 \mathrm{~km} \mathrm{~s}^{-1}$ and from 


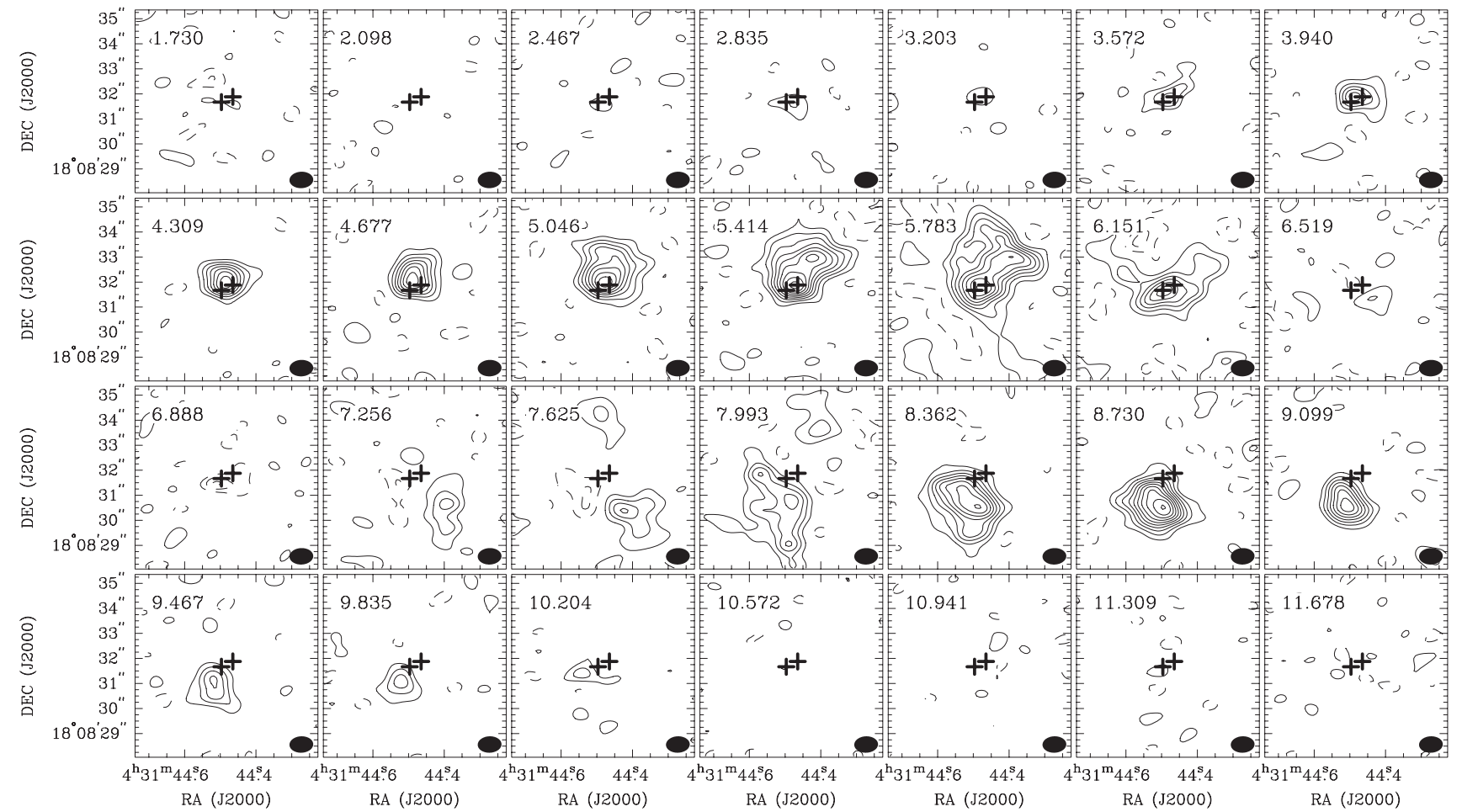

Figure 3. Velocity channel maps of the ${ }^{13} \mathrm{CO}(3-2)$ line in L1551 NE observed with the SMA. Contour levels are from $2 \sigma$ in steps of $2 \sigma\left(1 \sigma=0.107 \mathrm{Jy}\right.$ beam ${ }^{-1}$ ). Crosses indicate the positions of the protobinary, and a filled ellipse at the bottom-right corner in each panel indicates the synthesized beam $\left(0{ }^{\prime \prime} \cdot 95 \times 00^{\prime \prime} 66\right.$; P.A. $=$ $-88^{\circ}$ ). A number at the top-left corner in each panel denotes the LSR velocity.

$\sim 8.7$ to $9.5 \mathrm{~km} \mathrm{~s}^{-1}$ almost $100 \%$ of the total $\mathrm{C}^{18} \mathrm{O}(3-2)$ flux is recovered in our SMA observations, although the limited S/N of the single-dish spectrum at the line wings prevents us from accurately estimating the amount of the recovered flux. Thus, the systematic increase in the spatial extent of the $\mathrm{C}^{18} \mathrm{O}(3-2)$ emission over these velocity ranges, where the detection level is more than $6 \sigma$ except for that at the bluest and the reddest velocities, is probably real.

To better highlight the spatial-kinematic distributions of the different features traced by the ${ }^{13} \mathrm{CO}$ and $\mathrm{C}^{18} \mathrm{O}$ emission, we integrated these velocity channel maps over the four different velocity ranges discussed above, that is, highblueshifted $\left(2.5 \mathrm{~km} \mathrm{~s}^{-1}-3.6 \mathrm{~km} \mathrm{~s}^{-1}\right)$ and redshifted velocities $\left(9.8 \mathrm{~km} \mathrm{~s}^{-1}-10.2 \mathrm{~km} \mathrm{~s}^{-1}\right)$ where only ${ }^{13} \mathrm{CO}$ emission is detected, and low-blueshifted $\left(3.9 \mathrm{~km} \mathrm{~s}^{-1}-5.8 \mathrm{~km} \mathrm{~s}^{-1}\right)$ and redshifted velocities $\left(7.6 \mathrm{~km} \mathrm{~s}^{-1}-9.5 \mathrm{~km} \mathrm{~s}^{-1}\right)$ where $\mathrm{C}^{18} \mathrm{O}$ emission is also detected (Figure 5). Overall, the low-velocity blueshifted and redshifted components in the ${ }^{13} \mathrm{CO}$ and $\mathrm{C}^{18} \mathrm{O}$ maps are located to the northwest and southeast, respectively, of the L1551 NE system. Furthermore, the axis connecting the blueshifted and redshifted emission peaks is approximately perpendicular to the axis of the [Fe II] jets driven by Source A (see blue and red arrows in Figure 5). Although the symmetric center of the blueshifted and redshifted emission appears to be closer to the position of Source A, pinpointing the precise location of the symmetric center may not be straightforward as it seems. A comparison with the $\mathrm{CSO} \mathrm{C}^{18} \mathrm{O}(3-2)$ spectrum indicates that the amount of the missing flux at the blueshifted part of the SMA $\mathrm{C}^{18} \mathrm{O}$ emission is more than that at the redshifted part (for example, at $V_{\text {sys }}-1.0 \mathrm{~km} \mathrm{~s}^{-1}$ the amount of the missing flux is $\sim 80 \%$, while at $V_{\text {sys }}+1.0 \mathrm{~km} \mathrm{~s}^{-1} \sim 60 \%$ ). Furthermore, the location of L1551 NE overlaps with the spatially extended redshifted $\left(\sim 7-\sim 12 \quad \mathrm{~km} \mathrm{~s}^{-1}\right)$ outflow driven by L1551 IRS 5
(Moriarty-Schieven et al. 2006). Thus the redshifted part of the ${ }^{13} \mathrm{CO}$ emission may be more severely affected by missing flux than the blueshifted part. These "asymmetric" contributions from the extended components may distort the "balance" between the blueshifted and redshifted emission distributions.

In the high-velocity range, the compact $(\sim 100$ AU) blueshifted and redshifted ${ }^{13} \mathrm{CO}$ components are located to the northwest and southeast of Source A, respectively. The position angle of the axis connecting the high-velocity blueshifted and redshifted ${ }^{13} \mathrm{CO}$ components is different from that of the lowvelocity components; specifically, this axis is not orthogonal to the axis of the $[\mathrm{Fe}$ II] jets driven by Source A. These results imply that the origin of these high-velocity ${ }^{13} \mathrm{CO}$ components is different from that of the low-velocity components.

Figure 6 shows intensity-weighted mean-velocity maps of the low-velocity ${ }^{13} \mathrm{CO}$ and $\mathrm{C}^{18} \mathrm{O}$ emission. As mentioned above, the low-velocity blueshifted emission is distributed northwest and redshifted emission southeast of the L1551 NE system. We do not detect a velocity gradient along the minor axis of this structure indicative of an inflow, or contamination by an outflow. Thus, both the spatial and kinematic major axes of this feature are approximately perpendicular to the axis of the associated $[\mathrm{Fe}$ II] jets (blue and red arrows in Figure 6).

\section{ANALYSIS}

As shown in the previous section, the ${ }^{13} \mathrm{CO}(3-2)$ and $\mathrm{C}^{18} \mathrm{O}$ (3-2) emission immediately surrounding L1551 NE exhibit a conspicuous velocity gradient along their major axes perpendicular to the axis of the [Fe II] jets driven by Source A. Such a velocity gradient could be produced by either a purely rotating or a rotating and contracting (i.e., infalling component) disk. To differentiate between these possibilities, we tried model fittings 


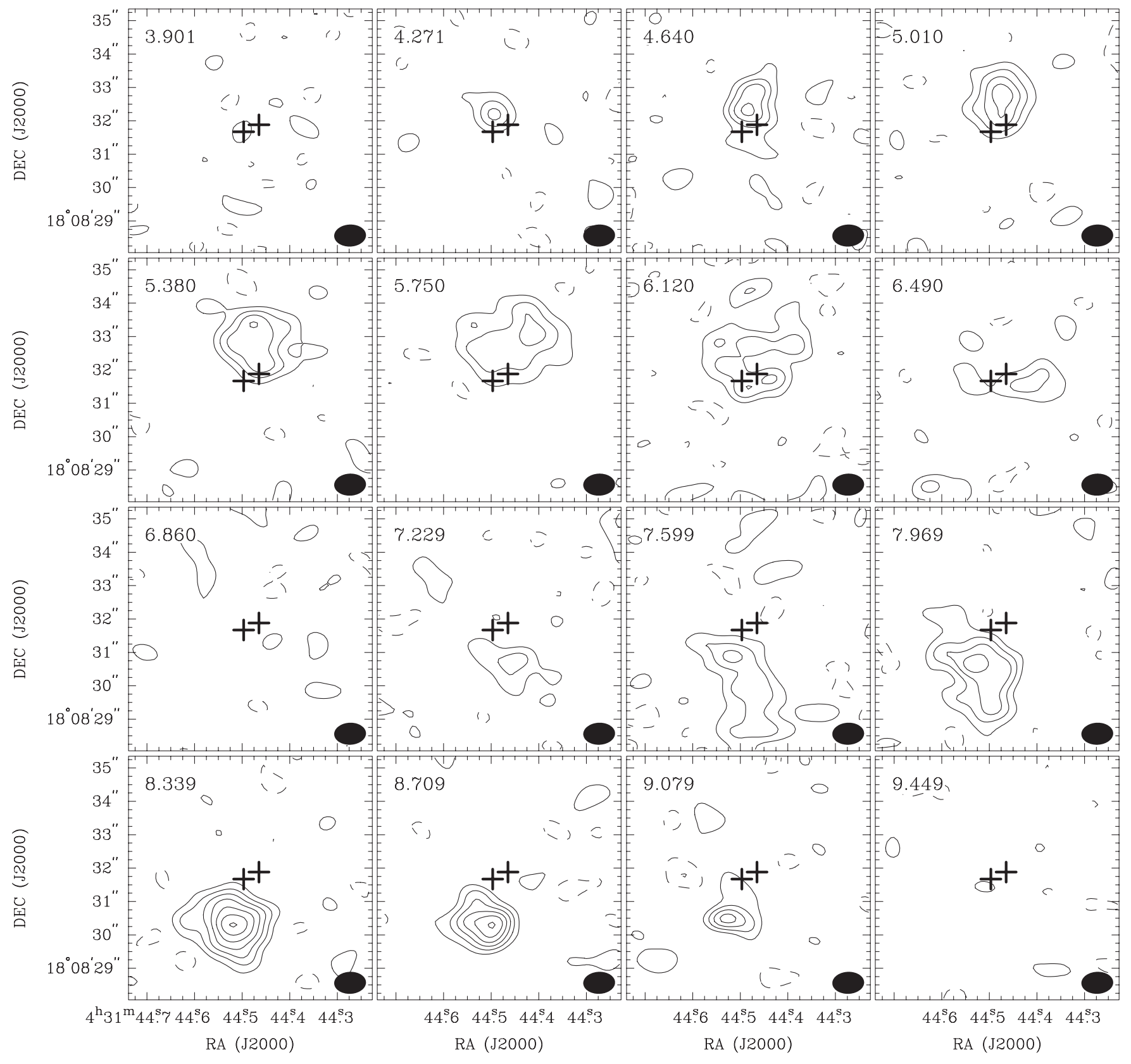

Figure 4. Velocity channel maps of the $\mathrm{C}^{18} \mathrm{O}(3-2)$ line in L1551 NE observed with the SMA. Contour levels are from $2 \sigma$ in steps of $2 \sigma\left(1 \sigma=0.117 \mathrm{Jy}\right.$ beam ${ }^{-1}$ ). Crosses indicate the positions of the protobinary, and a filled ellipse at the bottom-right corner in each panel indicates the synthesized beam $\left(00^{\prime \prime} 95 \times 00^{\prime \prime} 66 ;\right.$ P.A. $=$ $-88^{\circ}$ ). A number at the top-left corner in each panel denotes the LSR velocity.

of geometrically thin Keplerian or infalling disks to the observed ${ }^{13} \mathrm{CO}$ and the $\mathrm{C}^{18} \mathrm{O}$ channel maps.

Since we assume that the disk is geometrically thin, the centroid line-of-sight velocity at each position in right ascension and declination with respect to the disk center $\left(\equiv v_{\mathrm{LOS}}(\alpha, \delta)\right)$ can be expressed as

$$
v_{\mathrm{LOS}}(\alpha, \delta)=v_{\text {sys }}+v_{\text {rot }}(r) \cos (\Phi-\theta)+v_{\text {rad }}(r) \sin (\Phi-\theta),
$$

where

$$
\begin{gathered}
\Phi=\arctan \left(\frac{\alpha}{\delta}\right), \\
r=\sqrt{\left(\frac{x}{\cos i}\right)^{2}+y^{2}},
\end{gathered}
$$

$$
\begin{aligned}
& x=\alpha \cos (\theta)-\delta \sin (\theta), \\
& y=\alpha \sin (\theta)+\delta \cos (\theta) .
\end{aligned}
$$

In the above expressions, $v_{\text {sys }}$ is the systemic velocity, $\theta$ is the position angle of the disk major axis, and $x$ and $y$ are coordinates of the disk along the minor and major axes, respectively. $v_{\text {rot }}(r)$ denotes the rotational velocity of the disk as a function of the radius $(\equiv r)$. In the case of Keplerian rotation, $v_{\text {rot }}(r)$ is expressed as

$$
v_{\mathrm{rot}}(r)=\sin i \sqrt{\frac{G M_{\star}}{r}},
$$

where $i$ is the inclination angle of the disk from the plane of the sky, $G$ is the gravitational constant, and $M_{\star}$ is the mass of 


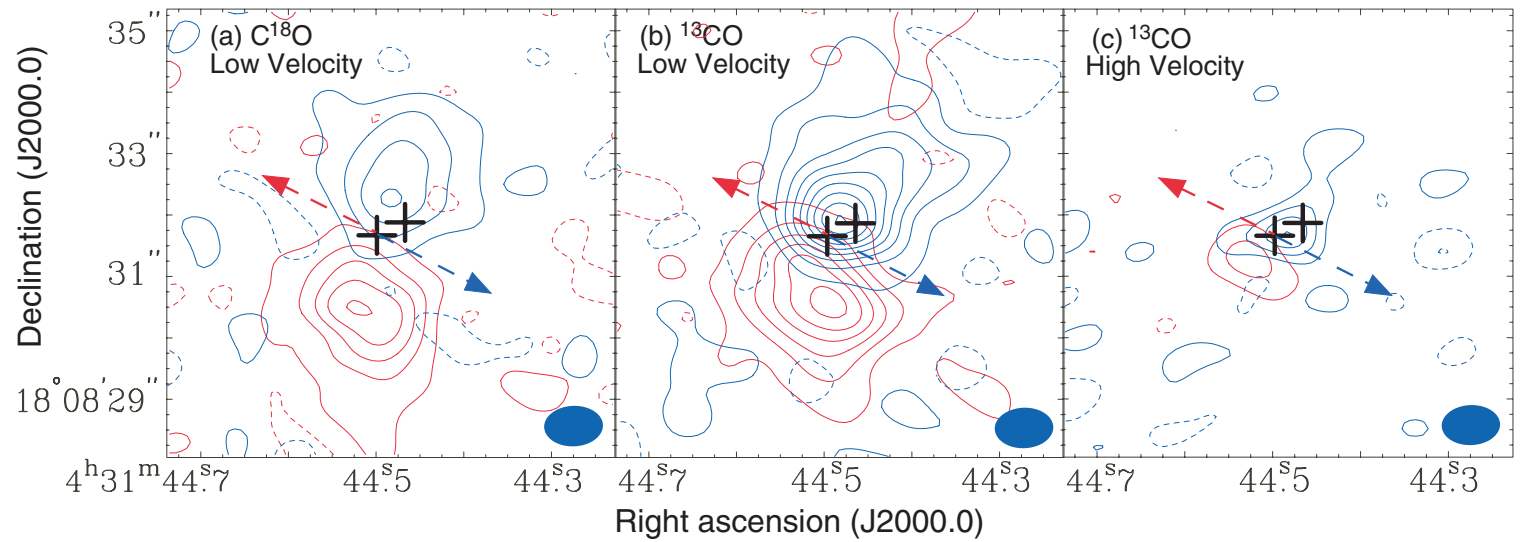

Figure 5. (a) Distributions of the low-velocity blueshifted (blue contours; $3.72-5.93 \mathrm{~km} \mathrm{~s}^{-1}$ ) and redshifted $\mathrm{C}^{18} \mathrm{O}(3-2)$ emission (red contours; 7.41-9.63 km s${ }^{-1}$ ) in L1551 NE observed with the SMA. Contour levels are from $0.212 \mathrm{Jy} \mathrm{beam}^{-1} \mathrm{~km} \mathrm{~s}^{-1}$ in steps of $0.424 \mathrm{Jy} \mathrm{beam}^{-1} \mathrm{~km} \mathrm{~s}^{-1}\left(1 \sigma=0.106 \mathrm{Jy} \mathrm{beam}{ }^{-1} \mathrm{~km} \mathrm{~s}^{-1}\right)$. Crosses indicate the positions of the protobinary, and a filled ellipse at the bottom-right corner indicates the synthesized beam $\left(0^{\prime \prime} 95 \times 00^{\prime \prime} 66 ;\right.$ P.A. $\left.=-88^{\circ}\right)$. Blue and red arrows show the direction of the blueshifted and redshifted $[\mathrm{Fe}$ II] jets driven by Source A. (b) Distributions of the low-velocity blueshifted (blue contours) and redshifted ${ }^{13} \mathrm{CO}(3-2)$ emission (red contours) in L1551 NE observed with the SMA. The integrated velocity ranges, contour levels, and the symbols are the same as those in (a), while the $1 \sigma \mathrm{rms}$ noise level is $0.097 \mathrm{Jy} \mathrm{beam}^{-1} \mathrm{~km} \mathrm{~s}^{-1}$. (c) Distributions of the high-velocity blueshifted (blue contours; $2.24-3.72 \mathrm{~km} \mathrm{~s}{ }^{-1}$ ) and redshifted ${ }^{13} \mathrm{CO}(3-2)$ emission (red contours; $9.63-10.37 \mathrm{~km} \mathrm{~s}^{-1}$ ) in L1551 NE observed with the SMA. Contour levels are from $0.158 \mathrm{Jy} \mathrm{beam}^{-1} \mathrm{~km} \mathrm{~s}^{-1}$ in steps of $0.158 \mathrm{Jy}_{\text {beam }}^{-1} \mathrm{~km} \mathrm{~s}^{-1}$, where the $1 \sigma \mathrm{rms}$ noise levels are $0.079 \mathrm{Jy} \mathrm{beam}{ }^{-1} \mathrm{~km} \mathrm{~s}^{-1}$ and $0.056 \mathrm{Jy} \mathrm{beam}^{-1} \mathrm{~km} \mathrm{~s}^{-1}$ in the blueshifted and redshifted velocity ranges, respectively.

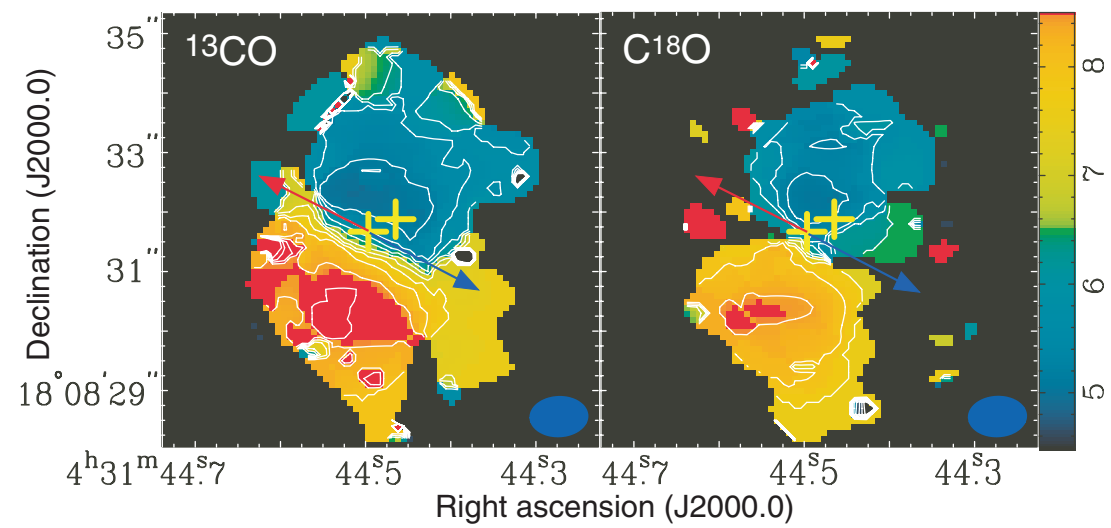

Figure 6. Moment 1 maps of the ${ }^{13} \mathrm{CO}(3-2)$ (left) and $\mathrm{C}^{18} \mathrm{O}$ (3-2) lines (right) in L1551 NE observed with the SMA. Contour levels are in steps of $0.4 \mathrm{~km} \mathrm{~s} \mathrm{~s}^{-1}$. The bluest and reddest contour levels to the northwest and southeast of Source $\mathrm{A}$ in the ${ }^{13} \mathrm{CO}$ map are $5.2 \mathrm{~km} \mathrm{~s}^{-1}$ and $8.8 \mathrm{~km} \mathrm{~s}^{-1}$, respectively, and those in the $\mathrm{C}^{18} \mathrm{O}$ map $5.2 \mathrm{~km} \mathrm{~s}^{-1}$ and $8.4 \mathrm{~km} \mathrm{~s}^{-1}$. Crosses indicate the positions of the protobinary, and filled ellipses at the bottom-right corners indicate the synthesized beam ( 0 '. $95 \times$ 0.'66; P.A. $=-88^{\circ}$ ). Blue and red arrows show the direction of the blueshifted and redshifted [Fe II] jets driven by Source A.

the central star. In the case of rotation with conserved angular momentum, which is expected for an infalling disk (Ohashi et al. 1997; Yen et al. 2011), $v_{\mathrm{rot}}(r)$ is expressed as

$$
v_{\text {rot }}(r)=\sin i \frac{j}{r},
$$

where $j$ denotes the specific angular momentum of the rotation. In the case of the infalling disk, radial motion $\left(\equiv v_{\text {rad }}(r)\right)$ is also present and can be expressed as

$$
v_{\mathrm{rad}}(r)=\sin i \sqrt{\frac{2 G M_{\star}}{r}} .
$$

The velocity channel maps of the model disk $\left(\equiv S_{\text {model }}(\alpha, \delta, v)\right)$ can be expressed as

$$
\begin{aligned}
S_{\text {model }}(\alpha, \delta, v)= & \left(S_{\text {mom0 } 0}(\alpha, \delta) / \sigma \sqrt{2 \pi}\right) \\
& \times \exp \left(\frac{-\left(v-v_{\mathrm{LOS}}(\alpha, \delta)\right)^{2}}{2.0 \sigma^{2}}\right),
\end{aligned}
$$

where $S_{\text {mom0 }}(\alpha, \delta)$ denotes the moment 0 map of the model disk and $\sigma$ the internal velocity dispersion.
In the model fitting, $S_{\text {momo } 0}(\alpha, \delta)$ was assumed to be the same as the observed ${ }^{13} \mathrm{CO}$ or $\mathrm{C}^{18} \mathrm{O}$ moment 0 maps (Figure 2). We adopted $\sigma$ to be $0.4 \mathrm{~km} \mathrm{~s}^{-1}$, inferred approximately from the ${ }^{13} \mathrm{CO}$ and $\mathrm{C}^{18} \mathrm{O}$ spectra. These assumptions mean that we fit only the global, systematic velocity structure in three-dimensional space, and that we do not fit the two-dimensional distributions of the molecular emission. The center of the model disk was fixed to be the position of Source A, and $v_{\text {sys }}$ was set to be $7 \mathrm{~km} \mathrm{~s}^{-1}$. The ${ }^{13} \mathrm{CO}$ and $\mathrm{C}^{18} \mathrm{O}$ velocity channel maps around the systemic velocity $\left(V_{\mathrm{LSR}}=6.52\right.$ and $6.89 \mathrm{~km} \mathrm{~s}^{-1}$ for ${ }^{13} \mathrm{CO}$ and $V_{\mathrm{LSR}}=6.86$ and $7.23 \mathrm{~km} \mathrm{~s}^{-1}$ for $\mathrm{C}^{18} \mathrm{O}$ ) were excluded from the fitting, since in these velocities the bulk of the emission is "resolved out." On these assumptions, we conducted minimum $\chi^{2}$-fittings of the model Keplerian disk and the model infalling disk to the observed ${ }^{13} \mathrm{CO}$ and $\mathrm{C}^{18} \mathrm{O}$ velocity channel maps, with $M_{\star}, \theta, i$, and $j$ (in the case of the infalling disk) as fitting parameters:

$$
\chi^{2}=\sum_{\alpha, \delta, v}\left(\frac{S_{\mathrm{obs}}(\alpha, \delta, v)-S_{\text {model }}^{M_{\star}, \theta, i, j}(\alpha, \delta, v)}{\sigma_{\mathrm{rms}}}\right)^{2} / \sum_{\alpha, \delta, v},
$$



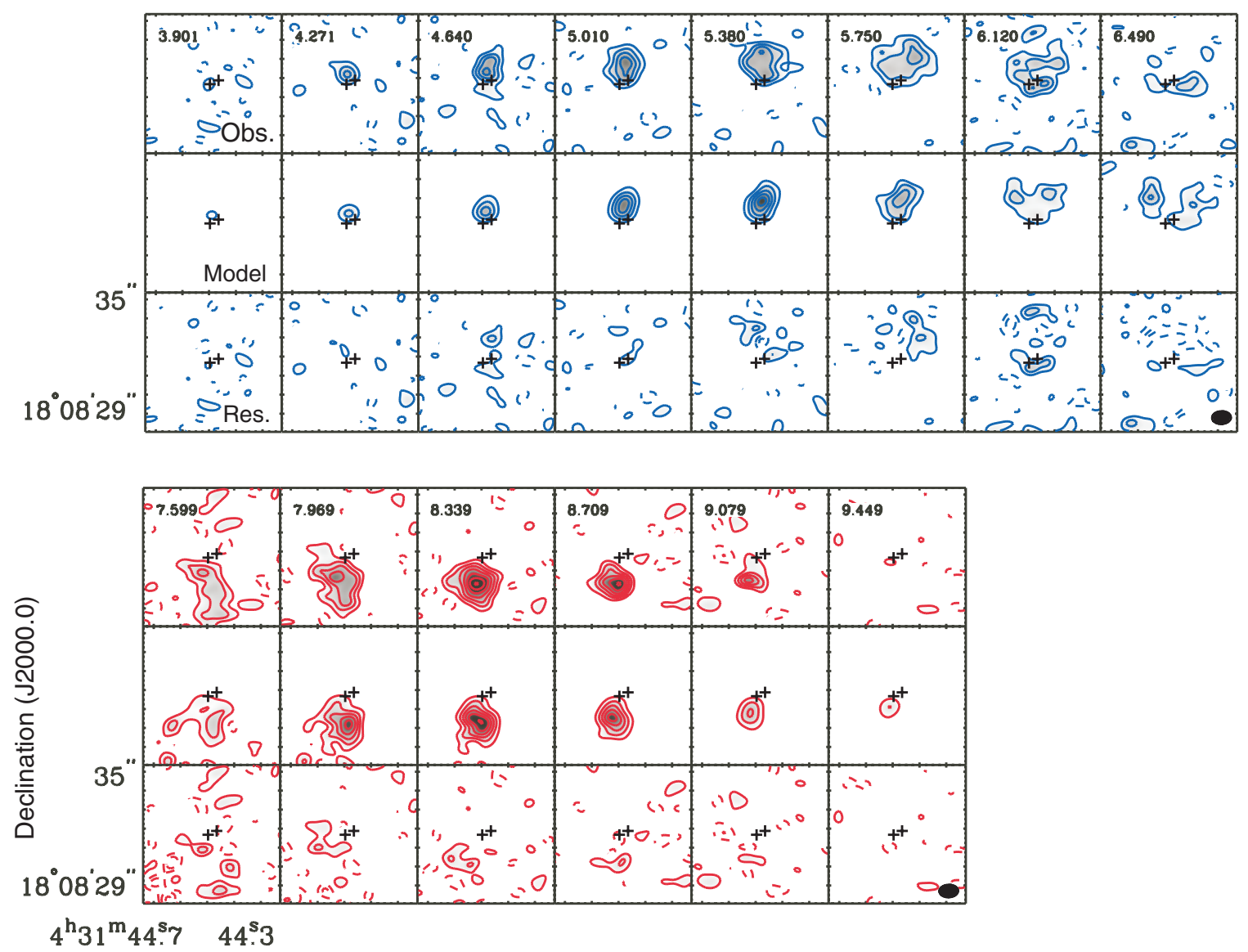

Right ascension (J2000.0)

Figure 7. Result of the $\chi^{2}$ fitting of the geometrically thin Keplerian disk to the $\mathrm{C}^{18} \mathrm{O}(3-2)$ velocity channel maps in L1551 NE at the blueshifted (blue contours) and redshifted (red contours) velocities. Upper, middle, and lower panels show the observed, model, and the residual velocity channel maps, respectively, where the best-fit parameters are $M_{\star}=0.8 M_{\odot}, \theta=167^{\circ}$, and $i=-62^{\circ}$. Contour levels are from $2 \sigma$ in steps of $2 \sigma\left(1 \sigma=0.117 \mathrm{Jy}\right.$ beam $\left.{ }^{-1}\right)$. Crosses show the position of the protobinary, and the filled ellipses at the bottom-right corners indicate the SMA synthesized beam $\left(0^{\prime \prime} .95 \times 00^{\prime \prime} 66 ;\right.$ P.A. $\left.=-88^{\circ}\right)$.

where $S_{\text {obs }}(\alpha, \delta, v)$ denotes the observed velocity channel maps, $S_{\text {model }}^{M_{\star}, \theta, j}(\alpha, \delta, v)$ the model velocity channel maps with a given $M_{\star}, \theta, i$, and $j$, and $\sigma_{\text {rms }}$ is the rms noise level of the observed velocity channel maps.

The middle and lower panels of Figure 7 show the best-fit Keplerian-disk model and the residual velocity channel maps of the $\mathrm{C}^{18} \mathrm{O}$ emission, respectively, where the best-fit parameters are $M_{\star}=0.8_{-0.4}^{+0.6} M_{\odot}, \theta=167_{-27^{\circ}}^{\circ}$, and $i=-62_{-17^{\circ}}^{\circ}$. The residual velocity channel maps do not show any systematic features although there are occasional $4 \sigma$ peaks; such random features with $4 \sigma$ peaks are also seen in the Keplerian model to DM Tau by Guilloteau \& Dutrey (1998). The best-fit residual $\mathrm{rms}$ is $\sim 0.144 \mathrm{Jy} \mathrm{beam}^{-1}$, which is slightly higher than but comparable to the noise level of the observed $\mathrm{C}^{18} \mathrm{O}$ velocity channel maps $\left(1 \sigma \sim 0.117 \mathrm{Jy}\right.$ beam $\left.^{-1}\right)$. Our model fit of an infalling disk to the $\mathrm{C}^{18} \mathrm{O}$ velocity channel maps, on the other hand, cannot find the local $\chi^{2}$ minimum within the range of $\theta$ from $109^{\circ}$ to $199^{\circ}$; i.e., the disk major axis is approximately perpendicular to the axis of the [Fe II] jets and more than $45^{\circ}$ away from the jet axis. These results show that a simple geometrically thin Keplerian-disk model with only three fitting parameters reproduces the global velocity feature in the $\mathrm{C}^{18} \mathrm{O}$ velocity channel maps. The position angle of the Keplerian rotation is consistent with the major axis of the $\mathrm{C}^{18} \mathrm{O}$ total integrated intensity map, and is approximately perpendicular to the axis of the [Fe II] jets driven by Source A. The estimated inclination angle of the disk from the plane of the sky is also approximately perpendicular to the inferred inclination angle of the [Fe II] jets of $i=30^{\circ}-45^{\circ}$ (Hayashi \& Pyo 2009). The position angle and the extent of the Keplerian disk in the $\mathrm{C}^{18} \mathrm{O}$ emission are similar to those of the ring-like structure in the $335 \mathrm{GHz}$ continuum emission surrounding the binary protostars. These results show that the circumbinary continuum feature is indeed a Keplerian disk surrounding the L1551 NE binary system, or "Keplerian circumbinary disk." Interestingly, the estimated position and inclination angles of the circumbinary disk around L1551 NE are similar to those of the circumstellar disks (Lim \& Takakuwa 2006) and the protostellar envelope (Momose et al. 1998; Takakuwa et al. 2004) around L1551 IRS 5 , but their rotation is in the opposite directions (in L1551 IRS 5 the northern part is redshifted).

By contrast, we could not find a satisfactory Keplerian fit (the peak residual is more than $10 \sigma$ ) to the ${ }^{13} \mathrm{CO}$ velocity channel maps. The same Keplerian-disk parameters do reproduce the overall spatial-velocity distributions in the ${ }^{13} \mathrm{CO}$ velocity channel maps, albeit with large residuals, presumably because the ${ }^{13} \mathrm{CO}$ emission is optically thick and likely affected by the contamination from the extended envelope component and possibly the outflow from L1551 IRS 5. Our simple geometrically thin disk model, which implicitly assumes optically thin emission, therefore cannot be accurately applied for the ${ }^{13} \mathrm{CO}$ emission. 


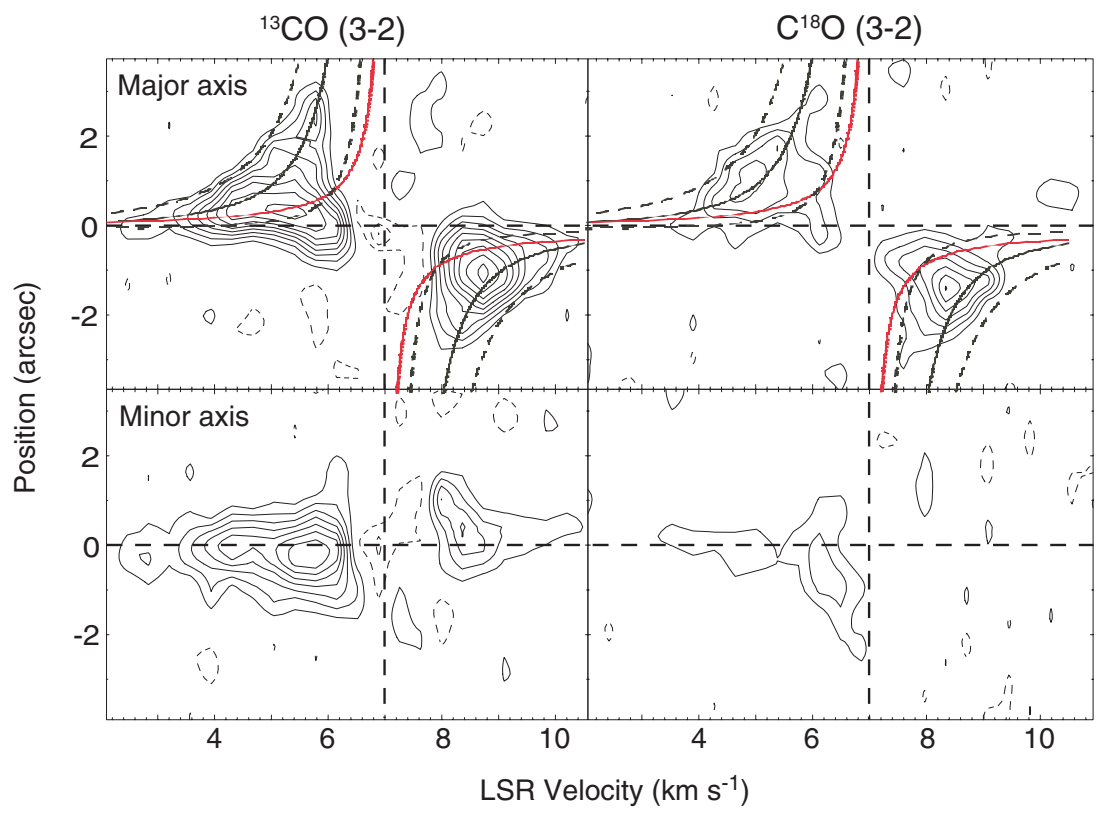

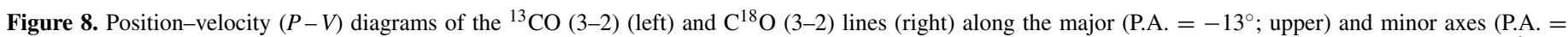

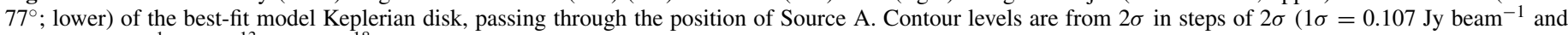

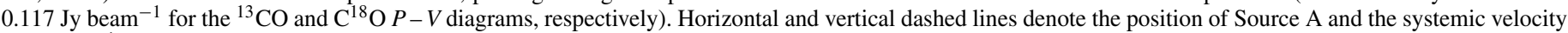

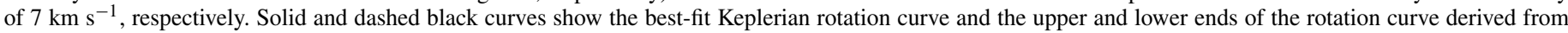
the error bars of the fitting parameters, while red curves denote rotation with the conserved specific angular momentum expected in infalling gas (see the text).

Figure 8 shows position-velocity $(P-V)$ diagrams of the ${ }^{13} \mathrm{CO}$ (left panels) and $\mathrm{C}^{18} \mathrm{O}$ emission (right) along the major (upper) and minor axes (lower) of the model Keplerian disks, passing through the location of Source A. In the $P-V$ diagrams along the major axis, the blueshifted and redshifted components are distinctly separated and located to the north and south, respectively, of L1551 NE. The emission at higher velocities is located closer to Source A than that at lower velocities, with this trend more pronounced in the ${ }^{13} \mathrm{CO}$ line. The bestfit Keplerian-rotation curve is shown overlaid as solid black curves, and the upper and lower ends of the rotational velocity derived from the error bars of the fitting parameters from the $\chi^{2}$-fitting overlaid as dashed black curves. For comparison, a rotation curve with a conserved angular momentum as expected for infalling gas $\left(j=6 \times 10^{-4} \mathrm{pc} \mathrm{km} \mathrm{s}^{-1}\right.$; red curve) is also plotted. Although both black and red curves trace the highervelocity parts close to the center $\left(V_{\mathrm{LSR}} \sim 2.5-4.5 \mathrm{~km} \mathrm{~s}^{-1}\right.$ and 9-10 $\mathrm{km} \mathrm{s}^{-1}$ ) equally well, the Keplerian-rotation curve better traces the lower-velocity parts $\left(\sim 5-6 \mathrm{~km} \mathrm{~s}^{-1}\right.$ and $\left.8-9 \mathrm{~km} \mathrm{~s}^{-1}\right)$. We note that the effect of the missing flux may distort the true rotation curves. Keplerian-rotation curves $\left(v_{\text {rot }} \propto r^{-0.5}\right)$ are, however, shallower than rotation curves with conserved angular momenta $\left(v_{\text {rot }} \propto r^{-1}\right)$, and thus the spatial extent of the Keplerian disk at a given velocity is larger, as shown by the black and red curves. Hence, it is possible that a genuine Keplerian rotation mimics a $r^{-1}$ rotation due to the effects of the missing flux, but it is unlikely that the effect of the missing flux distorts a genuine $r^{-1}$ rotation to a Keplerian rotation.

\section{DISCUSSION}

\subsection{Evolution of Disks around Protostars}

Our SMA observations of L1551 $\mathrm{NE}$ in the ${ }^{13} \mathrm{CO}$ and $\mathrm{C}^{18} \mathrm{O}(3-2)$ lines have revealed a Keplerian disk around the protobinary system. From the outer extent of the molecular-line distributions, the outermost detectable radius of the Keplerian disk is estimated to be $\sim 300 \mathrm{AU}$. The ring-like component seen in the $335 \mathrm{GHz}$ continuum emission likely traces this Keplerian circumbinary disk, while the central component may arise from circumstellar disks around the binary companions. The central mass derived from the Keplerian fitting $\left(\sim 0.8 M_{\odot}\right)$ is likely the mass of the protobinary, since the mass of the Keplerian circumbinary disk plus circumstellar disks derived from the $335 \mathrm{GHz}$ continuum emission $\left(\sim 0.047 M_{\odot}\right)$ is much smaller.

Previous interferometric observations of Class 0 protostars have revealed infalling motion in compact $(\lesssim 600 \mathrm{AU})$ circumstellar structures, i.e., pseudo-disks (Takakuwa et al. 2007; Brinch et al. 2009; Yen et al. 2010, 2011). On the other hand, the large SMA survey for protostellar sources ("PROSAC" project) has found Keplerian disks in the $\mathrm{HCO}^{+}(3-2)$ line around four Class I protostars (L1489-IRS, IRS 63, Elias 29, and IRS 43) out of their ten Class I samples (Brinch et al. 2007; Lommen et al. 2008; Jørgensen et al. 2009), one of which (L1489-IRS) was reported as a protobinary (Hogerheijde \& Sandell 2000). In the other six Class I sources the velocity fields traced by the $\mathrm{HCO}^{+}(3-2)$ line are rather complicated, presumably due to the contamination from the outflows. The reported Keplerian disks have radii $\sim 100-200 \mathrm{AU}$ and masses $\leqslant 0.007$ to $0.055 M_{\odot}$, and enclosed stellar masses $0.37-2.5 M_{\odot}$. These results imply that Keplerian disks can be present around Class I protostars as well as T Tauri stars (e.g., Guilloteau \& Dutrey 1998; Guilloteau et al. 1999). The measured physical properties of these protostars and the Keplerian disks, including those of L1551 NE, are summarized in Table 2.

Table 2 shows that there are no clear differences of the disk radius and the enclosed stellar mass between L1551 NE and the other sources. On the other hand, the bolometric temperature of L1551 NE $\left(T_{\mathrm{bol}}=91 \mathrm{~K}\right)$ is a factor $\gtrsim 3$ lower than that of the other protostars, and the mass of the surrounding envelope around L1551 NE is a factor of $\sim 4$ higher than that of L1489IRS and over an order of magnitude higher than that around the other protostars. Furthermore, the fraction of the central 
Table 2

Protostellar Sources with Keplerian Disks

\begin{tabular}{lcccccccc}
\hline \hline Source & $\begin{array}{c}L_{\text {bol }} \\
\left(L_{\odot}\right)\end{array}$ & $\begin{array}{c}T_{\text {bol }} \\
(\mathrm{K})\end{array}$ & $\begin{array}{c}R_{\text {kep }}{ }^{\mathrm{a}} \\
(\mathrm{AU})\end{array}$ & $\begin{array}{c}M_{\text {star }}{ }^{\mathrm{b}} \\
\left(M_{\odot}\right)\end{array}$ & $\begin{array}{c}M_{\text {disk }}{ }^{\mathrm{c}} \\
\left(M_{\odot}\right)\end{array}$ & $\begin{array}{c}M_{\text {env }}{ }^{\mathrm{d}} \\
\left(M_{\odot}\right)\end{array}$ & $\begin{array}{c}\frac{M_{\text {star }}}{M_{\text {star }}+M_{\text {disk }}+M_{\text {env }}} \\
(\%)\end{array}$ & $\begin{array}{c}\text { References } \\
\text { e }\end{array}$ \\
\hline L1551 NE & 4.2 & 91 & 300 & 0.8 & $0.026^{\mathrm{f}}$ & 0.39 & 65 & $1,2,3$ \\
L1489-IRS & 3.7 & 238 & 200 & 1.35 & 0.004 & 0.093 & 93 & 4,5 \\
IRS 43 & 6.0 & 310 & 140 & 1.0 & 0.0081 & 0.026 & 97 & 5 \\
IRS 63 & 0.79 & 351 & 100 & 0.37 & 0.055 & 0.022 & 83 & 5,6 \\
Elias 29 & 13.6 & 391 & 200 & 2.5 & $\leqslant 0.007$ & 0.025 & 399 & 5,6 \\
\hline
\end{tabular}

Notes.

a Outer radius of the Keplerian disk.

b Central protostellar mass derived from the Keplerian-disk model fitting.

${ }^{c}$ Mass of the Keplerian disk measured from the SMA continuum flux.

${ }^{\mathrm{d}}$ Mass of the protostellar envelope estimated from the single-dish submillimeter continuum flux.

e References: (1) This work; (2) Froebrich 2005; (3) Moriarty-Schieven et al. 2006; (4) Brinch et al. 2007; (5) Jørgensen et al. 2009; (6) Lommen et al. 2008.

${ }^{\mathrm{f}}$ Excluding the mass of the central compact component that may arise from the circumstellar disks around the binary companions.

protostellar mass to the total (star + disk + envelope) mass is the lowest in L1551 NE (=65\%). These results imply that either L1551 NE will evolve into a much more massive star compared with the other protostars listed in Table 2, or L1551 NE is the youngest Class I protostar yet found to have a Keplerian disk. Since the L1551 region forms solar-type $\left(\lesssim 1 M_{\odot}\right)$ stars (Hayashi et al. 1993; Momose et al. 1998; Takakuwa et al. 2004; Hayashi \& Pyo 2009), the former case is unlikely. Thus, we suggest that L1551 NE has experienced "transition" from an infalling, pseudo-disk to a Keplerian disk most recently among the Class I sources with the Keplerian disks. The formation mechanism of Keplerian disks around protostars will be discussed in the next section.

\subsection{On the Formation of a Keplerian Circumbinary Disk}

Our SMA observations of L1551 NE provide firm evidence that Keplerian circumbinary disks are present in addition to $\mathrm{Ke}$ plerian circumstellar disks around Class I protostars. On the theoretical side, however, it is still difficult to form Keplerian disks around either single or multiple protostars. Mellon \& Li (2008, 2009) have conducted two-dimensional axisymmetric simulations of the collapse of rotating, magnetized singular isothermal cores. They found that, even in the case of weak initial magnetic fields (mass-to-flux ratio $\lambda \lesssim 100$ ), magnetic fields efficiently carry angular momenta outward, preventing the formation of a rotationally supported disk. They showed that a field strength in the dense core L1544 inferred observationally $(\lambda \lesssim 10$; Crutcher $\&$ Troland 2000) completely suppresses the formation of the centrifugally supported disk, even if the ambipolar diffusion is taken into account. They concluded that additional processes, such as Ohmic dissipation, Hall effect, or the dispersion of the surrounding envelope that anchors the magnetic brake, are required for a Keplerian disk to form. Machida et al. (2010, 2011) and Machida \& Matsumoto (2011) have performed three-dimensional magnetohydrodynamic simulations of a Bonnor-Ebert sphere with a mass of $1 M_{\odot}$ and different initial rotations and magnetic fields, and have calculated the evolutions. Their simulations show that the first adiabatic core formed prior to the formation of the protostar becomes a centrifugally supported disk with a size of $\gtrsim 100 \mathrm{AU}$ by the end of the calculations. Their results show that, however, the mass of the formed disk is considerably ( $\sim$ factor $2-100$ ) larger than that of the central protostar (see also Vorobyov 2009), and hence the formed disk is subject to further fragmentation. Further longterm simulations, including calculations of fragmentations of the massive disks and subsequent evolutions, are required to theoretically reproduce the observed Keplerian disks around the Class I protostars.

Although a Keplerian circumbinary disk is identified around L1551 NE, L1551 NE is associated with active jets (Reipurth et al. 2000; Hayashi \& Pyo 2009) and molecular outflows (Moriarty-Schieven et al. 1995a), suggesting presence of accreting circumstellar disks around the binary companions. The central compact dust emission (Figure 1) may indeed arise from the circumstellar disks. Simulations of fragmentations of a first core or a massive disk into multiple protostellar systems (i.e., Machida et al. 2008) and further evolutions are required to reproduce a Keplerian circumbinary disk plus accreting circumstellar disks in a protobinary system. In the case of binary protostars, tidal effects from the two protostars can transport rotational angular momenta outward and create a "gap" in the circumbinary disk (Bate \& Bonnell 1997; Ochi et al. 2005). Furthermore, the inward transportation of magnetic fields in binary systems may be quite different from that around single protostars, because the mass accretion toward the binary protostars can occur through "spiral" accretion streams that connects between the inner edge of the circumbinary disk and the circumstellar disks (Bate \& Bonnell 1997; Ochi et al. 2005). The high-velocity ${ }^{13}$ CO components shown in Figure 5 may correspond to such accretion streams. If the angular momentum transported outward piles up at the inner edge of the circumbinary disk, this disk can then assume Keplerian rotation.

Finally, we will comment on an interesting application of Keplerian circumbinary disks. By measuring the position of the Keplerian rotation center with respect to the binary positions, in principle it is also possible to derive binary mass ratios, orbital radii, and orbital periods of binary protostars. Our $\chi^{2}$ model fitting of the geometrically thin Keplerian disk with the fixed rotational center at the position of Source A provides a reasonable fitting result. This result may suggest that the mass of Source A is higher than that of Source B, although our search for the minimum $\chi^{2}$ point of the rotational center failed to obtain a statistically significant result (at the positions of Sources A and B the reduced $\chi^{2}$ values are $\sim 1.5$ and $\sim 1.8$, respectively.). If we could pinpoint the rotational center, the binary mass ratio can simply be estimated from the ratio of the distances from the rotational center to the primary and to the secondary. 
This is potentially a strong method to measure mass ratios of protostellar binaries, since previous multi-epoch ( $>5 \mathrm{yr}$ ), highresolution $\left(\sim 00^{\prime}\right.$. 1 ) observations of proper motions of protobinary systems could only deduce the reduced masses (Loinard et al. 2002; Rodríguez et al. 2003; Lim \& Takakuwa 2006). We can also measure the de-projected orbital radii assuming that the binary stars are in the same plane of the circumbinary disks. From the binary masses, mass ratios, and the de-projected orbital radii the orbital periods can also be obtained. These measurements could put stringent constraints on theories of binary formation.

\section{SUMMARY}

We have carried out subarcsecond resolution SMA observations of the protobinary system L1551 NE in the $335 \mathrm{GHz}$ continuum, ${ }^{13} \mathrm{CO}(3-2)$, and the $\mathrm{C}^{18} \mathrm{O}(3-2)$ emission. The main results are summarized below:

1. The $335 \mathrm{GHz}$ continuum emission in L1551 NE shows an intense central peak close to the protobinary, and secondary peaks to the north and south of the protobinary system located almost symmetrically with respect to the position of the protobinary. The central component may arise from circumstellar disks around each binary companion. The northern and southern components comprise a $\sim 600 \mathrm{AU}$ scale circumbinary disk, approximately perpendicular to the axis of the associated [Fe II] jets driven by Source A. The mass of the circumstellar disk is estimated to be $\sim 0.02-0.09 M_{\odot}$, and that of the circumbinary disk $\sim 0.03-0.12 M_{\odot}$.

2. The extent and position angle of the ${ }^{13} \mathrm{CO}(3-2)$ and $\mathrm{C}^{18} \mathrm{O}(3-2)$ emission are similar to those of the $335 \mathrm{GHz}$ continuum emission, although the locations of the emission peaks are different among the ${ }^{13} \mathrm{CO}, \mathrm{C}^{18} \mathrm{O}$, and the $335 \mathrm{GHz}$ emission. Both the ${ }^{13} \mathrm{CO}$ and $\mathrm{C}^{18} \mathrm{O}$ emission show a systematic velocity gradient along the major axis, which is perpendicular to the axis of the [Fe II] jets, such that their blueshifted and the redshifted emission are located to the north and south of the protostellar binary, respectively. In addition to this global velocity gradient, the high-velocity $\left(\gtrsim 3 \mathrm{~km} \mathrm{~s}^{-1}\right){ }^{13} \mathrm{CO}$ emission exhibits compact $(\sim 100 \mathrm{AU})$ blueshifted and redshifted components to the northwest and southeast of Source A with a different position angle from that of the circumbinary disk. We speculate that the highvelocity ${ }^{13} \mathrm{CO}$ components correspond to accretion streams that connects between the inner edge of the circumbinary disk and the circumstellar disks.

3. $\chi^{2}$ model fitting of geometrically thin Keplerian disks to the observed ${ }^{13} \mathrm{CO}$ and $\mathrm{C}^{18} \mathrm{O}$ velocity channel maps was conducted. The $\mathrm{C}^{18} \mathrm{O}$ velocity channel maps are well reproduced by a geometrically thin Keplerian-disk model with a central stellar mass of $0.8_{-0.4}^{+0.6} M_{\odot}$, disk position angle of $167^{\circ}+23^{\circ}$, and a disk inclination angle of $-62^{\circ+25^{\circ}}$, and a rotational center at the position of Source A. On the other hand, the ${ }^{13} \mathrm{CO}$ velocity channel maps cannot be fitted with any geometrically thin Keplerian-disk model. This is probably because the ${ }^{13} \mathrm{CO}$ emission is optically thick and is likely affected by the contamination from the outflow and the extended envelope component.

4. Among the Class I sources with identified Keplerian disks, L1551 NE is likely the youngest source, since L1551 NE has the lowest bolometric temperature $(\sim 91 \mathrm{~K})$, highest envelope mass $\left(\sim 0.39 M_{\odot}\right)$, and the lowest ratio in stellar mass to envelope + disk + stellar mass $(\sim 0.65)$. We suggest that L1551 NE has just passed from the "pseudo-disk" phase seen around Class 0 protostars to the Kepleriandisk phase. Our SMA observations of L1551 NE provide firm evidence that Keplerian disks around binary protostars, Keplerian circumbinary disks, are present, as well as Keplerian disks around single protostars.

5. Current theoretical studies cannot reproduce observationally identified Keplerian disks around single or multiple protostars. In the case of binary protostars, tidal effects from two protostars can transport rotational angular momenta outward, and pile up the angular momenta at the inner edge of the circumbinary disk effectively. Furthermore, the inward transportation of magnetic fields in binary systems may be quite different from that around single protostars, because the mass accretion toward the binary protostars can occur through "spiral" accretion streams from the inner edge of the circumbinary disk to the circumstellar disks. These mechanisms could create a Keplerian circumbinary disk around binary protostars. Higher-resolution observations of L1551 NE with ALMA and/or EVLA, which can separate between the circumstellar disks and the circumbinary disk and resolve the accretion streams connecting between the circumbinary disk and the circumstellar disks, are required to fully understand the origin of the Keplerian circumbinary disk plus the circumstellar disks.

We are grateful to P. T. P. Ho and N. Ohashi for their fruitful discussions. We thank all the SMA staff supporting this work. S.T. acknowledges a grant from the National Science Council of Taiwan (NSC 99-2112-M-001-009-MY3) in support of this work.

\section{REFERENCES}

Andrews, S. M., \& Williams, J. P. 2005, ApJ, 631, 1134

Andrews, S. M., \& Williams, J. P. 2007, ApJ, 671, 1800

Barsony, M., \& Chandler, C. J. 1993, ApJ, 406, L71

Bate, M. R., \& Bonnell, I. A. 1997, MNRAS, 285, 33

Beckwith, S. V. W., Sargent, A. I., Chini, R. S., \& Guesten, R. 1990, AJ, 99, 924

Brinch, C., Crapsi, A., Jørgensen, J. K., Hogerheijde, M. R., \& Hill, T. 2007, A\&A, 475, 915

Brinch, C., Jørgensen, J. K., \& Hogerheijde, M. R. 2009, A\&A, 502, 199

Chen, X., Arce, H. G., Dunham, M. M., \& Zhang, Q. 2012, ApJ, 747, L43

Crutcher, R. M., \& Troland, T. H. 2000, ApJ, 537, L139

Delfosse, X., Forveille, T., Beuzit, J.-L., et al. 1999, A\&A, 344, 897

Duquennoy, A., \& Mayor, M. 1991, A\&A, 248, 485

Elias, J. H. 1978, ApJ, 224, 857

Enoch, M. L., Corder, S., Duchêne, G., et al. 2011, ApJS, 195, 21

Enoch, M. L., Corder, S., Dunham, M. M., \& Duchêne, G. 2009, ApJ, 707, 103 Froebrich, D. 2005, ApJS, 156, 169

Fuller, G. A., \& Ladd, E. F. 2002, ApJ, 573, 699

Guilloteau, S., \& Dutrey, A. 1998, A\&A, 339, 467

Guilloteau, S., Dutrey, A., \& Simon, M. 1999, A\&A, 348, 570

Harvey, D. W. A., Wilner, D. J., Myers, P. C., \& Tafalla, M. 2003a, ApJ, 596, 383

Harvey, D. W. A., Wilner, D. J., Myers, P. C., Tafalla, M., \& Mardones, D. 2003b, ApJ, 583, 809

Hayashi, M., Ohashi, N., \& Miyama, S. M. 1993, ApJ, 418, L71

Hayashi, M., \& Pyo, T.-S. 2009, ApJ, 694, 582

Ho, P. T. P., Moran, J. M., \& Lo, K. Y. 2004, ApJ, 616, L1

Hogerheijde, M. R., \& Sandell, G. 2000, ApJ, 534, 880

Jørgensen, J. K., van Dishoeck, E. F., Visser, R., et al. 2009, A\&A, 507, 861

Lim, J., \& Takakuwa, S. 2006, ApJ, 653, 425

Loinard, L., Rodríguez, L. F., D’Alessio, P., Wilner, D. J., \& Ho, P. T. P. 2002, ApJ, 581, 109

Lommen, D., Jørgensen, J. K., van Dishoeck, E. F., \& Crapsi, A. 2008, A\&A, 481,141 
Looney, L. W., Mundy, L. G., \& Welch, W. J. 1997, ApJ, 484, L157

Looney, L. W., Mundy, L. G., \& Welch, W. J. 2000, ApJ, 529, 477

Machida, M. N., Inutsuka, S.-I., \& Matsumoto, T. 2010, ApJ, 724, 1006

Machida, M. N., Inutsuka, S.-I., \& Matsumoto, T. 2011, PASJ, 63, 555

Machida, M. N., \& Matsumoto, T. 2011, MNRAS, 413, 2767

Machida, M. N., Tomisaka, K., Matsumoto, T., \& Inutsuka, S.-i. 2008, ApJ, 677,327

Mathieu, R. D., Ghez, A. M., Jensen, E. L. N., \& Simon, M. 2000, in Protostars and Planets IV, ed. V. Mannings, A. P. Boss, \& S. S. Russell (Tucson, AZ: Univ. Arizona Press), 703

Maury, A. J., André, Ph., Hennebelle, P., et al. 2010, A\&A, 512, 40

Mellon, R. R., \& Li, Z.-Y. 2008, ApJ, 681, 1356

Mellon, R. R., \& Li, Z.-Y. 2009, ApJ, 698, 922

Momose, M., Ohashi, N., Kawabe, R., Nakano, T., \& Hayashi, M. 1998, ApJ, 504,314

Moriarty-Schieven, G. H., Butner, H. M., \& Wannier, P. G. 1995a, ApJ, 445, L55

Moriarty-Schieven, G. H., Johnstone, D., Bally, J., \& Jenness, T. 2006, ApJ, 645,357

Moriarty-Schieven, G. H., Powers, J. A., Butner, H. M., Wannier, P. G., \& Keene, J. 2000, ApJ, 533, L143

Moriarty-Schieven, G. H., Wannier, P. G., Keene, J., \& Tamura, M. 1994, ApJ, 436, 800

Moriarty-Schieven, G. H., Wannier, P. G., Mangum, J. G., Tamura, M., \& Olmsted, V. K. 1995b, ApJ, 455, 190
Ochi, Y., Sugimoto, K., \& Hanawa, T. 2005, ApJ, 623, 922

Ohashi, N., Hayashi, M., Ho, P. T. P., et al. 1997, ApJ, 488, 317

Ossenkopf, V., \& Henning, T. 1994, A\&A, 291, 943

Piétu, V., Dutrey, A., \& Kahane, C. 2003, A\&A, 398, 565

Reipurth, B., Rodríguez, L. F., Anglada, G., \& Bally, J. 2002, AJ, 124, 1045

Reipurth, B., Yu, K. C., Heathcote, S., Bally, J., \& Rodríguez, L. F. 2000, AJ, 120,1449

Rodríguez, L. F., Curiel, S., Cantó, J., et al. 2003, ApJ, 583, 330

Sault, R. J., Teuben, P. J., \& Wright, M. C. H. 1995, in ASP Conf. Ser. 77, Astronomical Data Analysis Software and Systems IV, ed. R. A. Shaw, H. E. Payne, \& J. J. E. Hayes (San Francisco, CA: ASP), 433

Schaefer, G. H., Dutrey, A., Guilloteau, S., Simon, M., \& White, R. J. 2009, ApJ, 701, 698

Scoville, N. Z., Carlstrom, J. E., Chandler, C. J., et al. 1993, PASP, 105, 1482

Takakuwa, S., \& Kamazaki, T. 2011, PASJ, 63, 921

Takakuwa, S., Ohashi, N., Bourke, T. L., et al. 2007, ApJ, 662, 431

Takakuwa, S., Ohashi, N., Ho, P. T. P., et al. 2004, ApJ, 616, L15

Visser, R., van Dishoeck, E. F., Doty, S. D., \& Dullemond, C. P. 2009, A\&A, 495,881

Vorobyov, E. I. 2009, ApJ, 692, 1609

Wilner, D. J., \& Welch, W. J. 1994, ApJ, 427, 898

Yen, H.-W., Takakuwa, S., \& Ohashi, N. 2010, ApJ, 710, 1786

Yen, H.-W., Takakuwa, S., \& Ohashi, N. 2011, ApJ, 742, 57

Yokogawa, S., Kitamura, Y., Momose, M., \& Kawabe, R. 2003, ApJ, 595, 266 